\title{
Biometrics via Spatial P-QRS-T Loop Features: Effect of Different VCG Transformations
}

\author{
Vessela Krasteva $^{1 *}$, Irena Jekova ${ }^{1}$, Ramun Schmid ${ }^{2}$ \\ ${ }^{1}$ Institute of Biophysics and Biomedical Engineering, Bulgarian Academy of Sciences, Sofia, Bulgaria \\ ${ }^{2}$ Signal Processing, Schiller AG, Baar, Switzerland
}

\begin{abstract}
The objective of biometrics is to identify subjects based on physiological or behavioral characteristics. This paper considers the spatial P-QRS-T loops of the vectorcardiogram (VCG), aiming to identify the most reliable $V C G$-based features for human verification. We analyze clinical standard 12-lead resting electrocardiograms (ECGs) from 460 non-cardiac patients with 2 recordings (>1 year apart). We build human verification models for nine ECG to VCG transformations. This study gives clear justification that VCG is applicable for human biometrics with true verification rate TVR=83.5-91.4\%. The 'Uijen' transformation has the best TVR for all VCG features (91.4\%) and individual features for P-loop (70.9\%), Tloop (77.1\%), QRS-loop (89.6\%), Frontal plane (84.5\%), Sagittal plane (84.1\%), 3D (91.1\%), while 'Dower' and 'Kors' are the best for the Horizontal plane (84.5\%).
\end{abstract}

\section{Introduction}

The objective of biometrics is to identify subjects based on physiological or behavioural characteristics, such as fingerprint, iris, face, voice, which however could easily be mimicked via fake finger, iris, face photos, playback, do not provide liveness detection [1] and are a topic for discussion on privacy protection [2]. The electrocardiogram (ECG) has been investigated as an advanced signal for human biometrics, presenting vital signs. The human verification or identification solutions employ a single ECG lead [3, 4], limb leads [5] or standard 12-lead ECG [6, 7], based on temporal and amplitude ECG features [6], cross-correlation analysis [5, 7], PQRST pattern matching [3, 4].

The spatial features of the cardiac vector represented by the vectorcardiogram (VCG) are expected to be useful for biometric applications, considering the inter-subject differences of the VCG loop orientation and shape, and its independence from the heart rate [8]. However, we could find a few studies based on VCG biometrics, all of them solving the human identification task, using:
- Support vector machine classifier, applied over QRSand T-loop features derived via inverse Dower transform [9] or pseudo-inverse transform, including only the limb leads [10];

- Neural networks classifier, applied over equal distance descriptor coefficients or Fourier descriptor coefficients of the QRS-loop constructed by plotting the QRS in lead I (x-axis) against lead aVF (y-axis), i.e. the QRS-loop projection in the vertical plane [8].

This paper considers the spatial P-QRS-T loops of the VCG, aiming to identify the most reliable VCG-based features for human verification. Presuming that different techniques for transformation of 12-lead ECG to VCG [11-13] and P-QRS-T loops projections in the Frontal, Horizontal and Sagittal planes [14] have specific diagnostic significance, we aim to compare their effect on human verification.

\section{ECG database}

The study is using a proprietary clinical ECG database (Schiller AG, Switzerland), which contains two 10ssessions of standard 12-lead resting ECGs from 460 noncardiac patients (235/225 male/female, 18-106 years old), admitted in the emergency department of the University Hospital Basel during the period (2004-2009). The ECGs are recorded via the commercial ECG device SCHILLER $(500 \mathrm{~Hz}, 2.5 \mu \mathrm{V} / \mathrm{LSB}$, bandwidth $0.05-150 \mathrm{~Hz})$ at distant time sessions $\mathrm{S} 1$ and $\mathrm{S} 2>\mathrm{S} 1+1$ year. The person verification scheme for comparison of subjects between $\mathrm{S} 1$ and $\mathrm{S} 2$ gives $\mathrm{N}=460$ pairs with equal identity (ID) and $N^{*}(\mathrm{~N}-1)=211140$ pairs with different ID. Our approach to handle the imbalance ratio (459:1) of different-to-equal ID pairs considers two independent datasets:

- Training dataset: 230/230 ECG pairs of equal/ different IDs, presuming that the verification classifier should be trained on the first half of subjects using balanced data, not over fitted to any of the classes.

- Test dataset: 230/210910 ECG pairs of equal/ different IDs, ensuring that unbiased classifier performance is further reported on a big dataset, including all available cases fully independent from the training. 


\section{Method}

\subsection{VCG transformations}

VCG is derived from the standard 12-lead ECG via inverse transform matrix Do, so that each of the orthogonal VCG leads (X, Y, Z) is a linear combination of the eight independent leads (I, II, V1-V6):

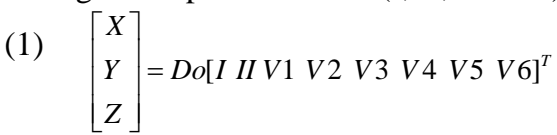

In Table 1 , the Do coefficients of nine established transformations are reproduced. Figure 1 compares all transformations, showing their Do coefficients in the orthogonal X,Y,Z space after normalization to the maximal Do value in each transformation.

\subsection{VCG features}

A commercial ECG measurement and interpretation module (ETM, Schiller AG) is used for beat averaging and delineation of ECG waves (Figure 2). These measurements are then used for the calculation of 71 spatial features of the beat-averaged VCG-loop:

- 3D features: 3 loops (P, QRS, T) x 8 features (circumference, width, area (value, azimuth, elevation), maximal vector (amplitude, azimuth, elevation)), spatial QRS-T angle, proportion of QRS area after the maximal vector.

- 2D features: 3 planes (Frontal (F), Horizontal (H), Sagittal (S)) $\times 3$ loops (P, QRS, T) x 5 features (circumference, width, area, maximal vector (amplitude, azimuth)).

\subsection{Human verification models}

The human verification answers the question: "Is the subject who he/she claims to be?". The verification model takes the binary decision 'verified' or 'rejected' subject ID, by comparing a pair of ECG recordings $\{\mathrm{S} 1, \mathrm{~S} 2\}$ with a linear discriminant analysis (LDA). The verification performance is estimated by the proportions of correctly verified equal $\mathrm{ID}_{\mathrm{S} 1}=\mathrm{ID}_{\mathrm{S} 2}$ (true acceptance rate, TAR), correctly rejected different $\mathrm{ID}_{\mathrm{S} 1} \neq \mathrm{ID}_{\mathrm{S} 2}$ (true rejection rate, $T R R$ ), and the common mean of TAR and TVR (true verification rate, $T V R)$ :

$$
\begin{aligned}
-T A R & =\frac{\text { Number Correct Verifications }}{\text { Nb Comparisons }\left(\mathrm{ID}_{\mathrm{S} 1}=\mathrm{ID}_{\mathrm{S} 2}\right)} \cdot 100,(\%) \\
-T R R & =\frac{\text { Number Correct Rejections }}{\text { Nb Comparisons }\left(\mathrm{ID}_{\mathrm{S} 1} \neq \mathrm{ID}_{\mathrm{S} 2}\right)} \cdot 100,(\%) \\
-T V R & =\frac{T A R+T R R}{2},(\%) \cdot
\end{aligned}
$$

Table 1. Inverse transform matrices $(3 \times 12)$ of standard 12-lead ECG to 3-lead VCG for nine published transformations. The largest coefficients in a row,

\begin{tabular}{|c|c|c|c|c|c|c|c|c|c|}
\hline & VCG & I & II & V1 & $\mathrm{V} 2$ & V3 & V4 & V5 & V6 \\
\hline Dower & $\mathrm{X}$ & 0.156 & -0.01 & -0.172 & -0.074 & 0.122 & 0.231 & 0.239 & 0.194 \\
\hline \multirow[t]{2}{*}{ [11] } & $\mathrm{Y}$ & -0.227 & 0.887 & 0.057 & -0.019 & -0.106 & -0.022 & 0.041 & 0.048 \\
\hline & Z & 0.022 & 0.102 & -0.229 & -0.31 & -0.246 & 0.063 & 0.055 & 0.108 \\
\hline Kors & $\mathrm{X}$ & 0.38 & -0.07 & -0.13 & 0.05 & -0.01 & 0.14 & 0.06 & 0.54 \\
\hline \multirow{2}{*}{ [11] } & $\mathrm{Y}$ & -0.07 & 0.93 & 0.06 & -0.02 & -0.05 & 0.06 & -0.17 & 0.13 \\
\hline & Z & 0.11 & -0.23 & -0.43 & -0.06 & -0.14 & -0.2 & -0.11 & 0.31 \\
\hline Uijen & $\mathrm{X}$ & 1.08 & -0.15 & -0.01 & 0.04 & 0.04 & 0.05 & 0.07 & 0.37 \\
\hline \multirow{2}{*}{ [11] } & Y & -0.325 & 1.31 & 0.03 & -0.02 & -0.02 & 0.03 & -0.07 & -0.08 \\
\hline & Z & -0.225 & -0.21 & -0.26 & -0.28 & -0.14 & 0.04 & -0.15 & 0.34 \\
\hline Willems & $\mathrm{X}$ & 0.82 & -0.24 & -1.27 & -0.55 & 0.72 & 1.86 & 1.92 & 1.53 \\
\hline \multirow[t]{2}{*}{ [11] } & Y & -3.04 & 5.62 & -0.71 & -0.71 & 0.1 & 0.35 & 0.12 & -0.15 \\
\hline & Z & 1.62 & -2.42 & -1.71 & -2.26 & -2.02 & -0.8 & 0.31 & 0.97 \\
\hline \multirow{3}{*}{$\begin{array}{c}\text { Bjerle } \\
{[11]}\end{array}$} & $\bar{X}$ & & & & & & & & 1.06 \\
\hline & Y & -0.625 & 1.25 & & & & & & \\
\hline & Z & & & & -0.532 & & & & 0.043 \\
\hline \multirow{3}{*}{$\begin{array}{c}\text { Arved- } \\
\text { son } \\
{[12]} \\
\end{array}$} & $\mathrm{X}$ & & & & & & & & 1.0625 \\
\hline & Y & -.5625 & 1.125 & & & & & & \\
\hline & Z & & & & -0.463 & & & & 0.037 \\
\hline \multirow{3}{*}{$\begin{array}{c}\text { Schreck } \\
\text { [11] }\end{array}$} & $\mathrm{X}$ & 1 & & & & & & & \\
\hline & $\mathrm{Y}$ & -0.5 & 1 & & & & & & \\
\hline & Z & & & & 1 & & & & \\
\hline \multirow{3}{*}{$\begin{array}{c}\text { Hyttinen } \\
\text { (Frank) } \\
{[11]}\end{array}$} & $\bar{X}$ & 0.357 & -0.519 & 0.605 & 0.087 & -0.002 & 0.167 & 0.306 & 0.192 \\
\hline & $\mathrm{Y}$ & 0.691 & -0.462 & 0.008 & 0.059 & -0.049 & 0.214 & -0.017 & 0.88 \\
\hline & Z & 0.2 & -0.946 & 0.043 & -0.017 & 0.017 & -0.032 & 0.237 & -0.204 \\
\hline \multirow{3}{*}{$\begin{array}{c}\text { Hyttinen } \\
\text { (VCG) } \\
{[11]} \\
\end{array}$} & $\bar{X}$ & 0.56 & 0.595 & 0.305 & 0.299 & -0.052 & -0.038 & 2.412 & -4.275 \\
\hline & $\mathrm{Y}$ & 1.245 & -0.563 & -0.13 & 0.053 & -0.001 & -0.072 & 1.004 & 0.217 \\
\hline & $\mathrm{Z}$ & 0.857 & -1.295 & 0.41 & -0.019 & 0.01 & -0.086 & 0.238 & -0.034 \\
\hline
\end{tabular}
corresponding to the significant ECG leads in the transformation are printed in bold.

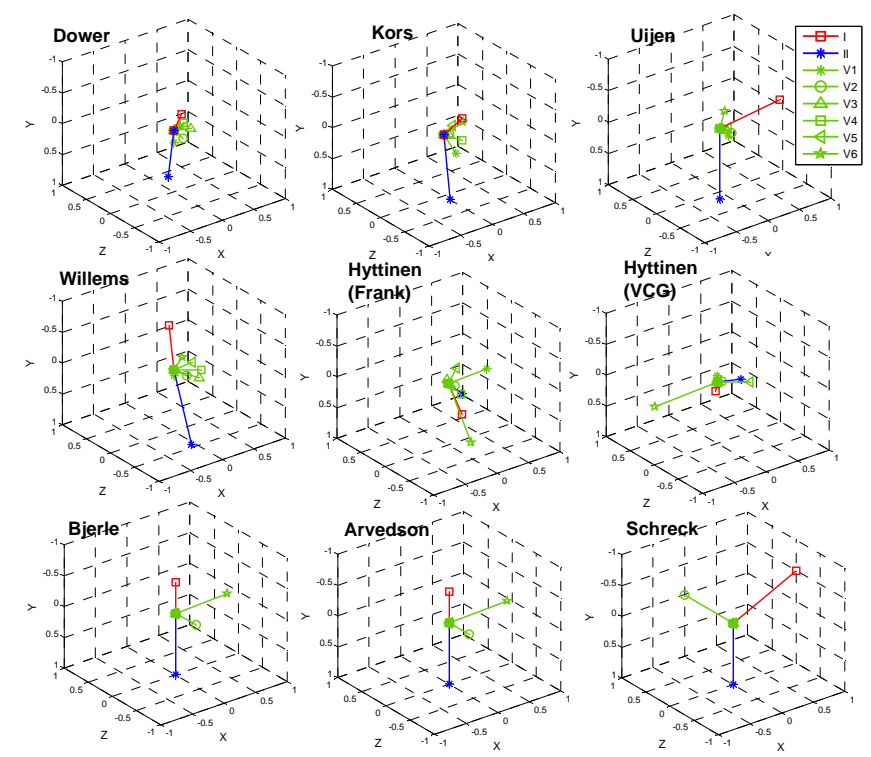

Figure 1. Comparison of VCG Transformations: 3D projection of Do coefficients (normalized to unity).

Non-redundant LDA models were trained by stepwise feature selection until TVR maximization, while keeping equal error rate $(T A R=T R R)$ on the training dataset. 

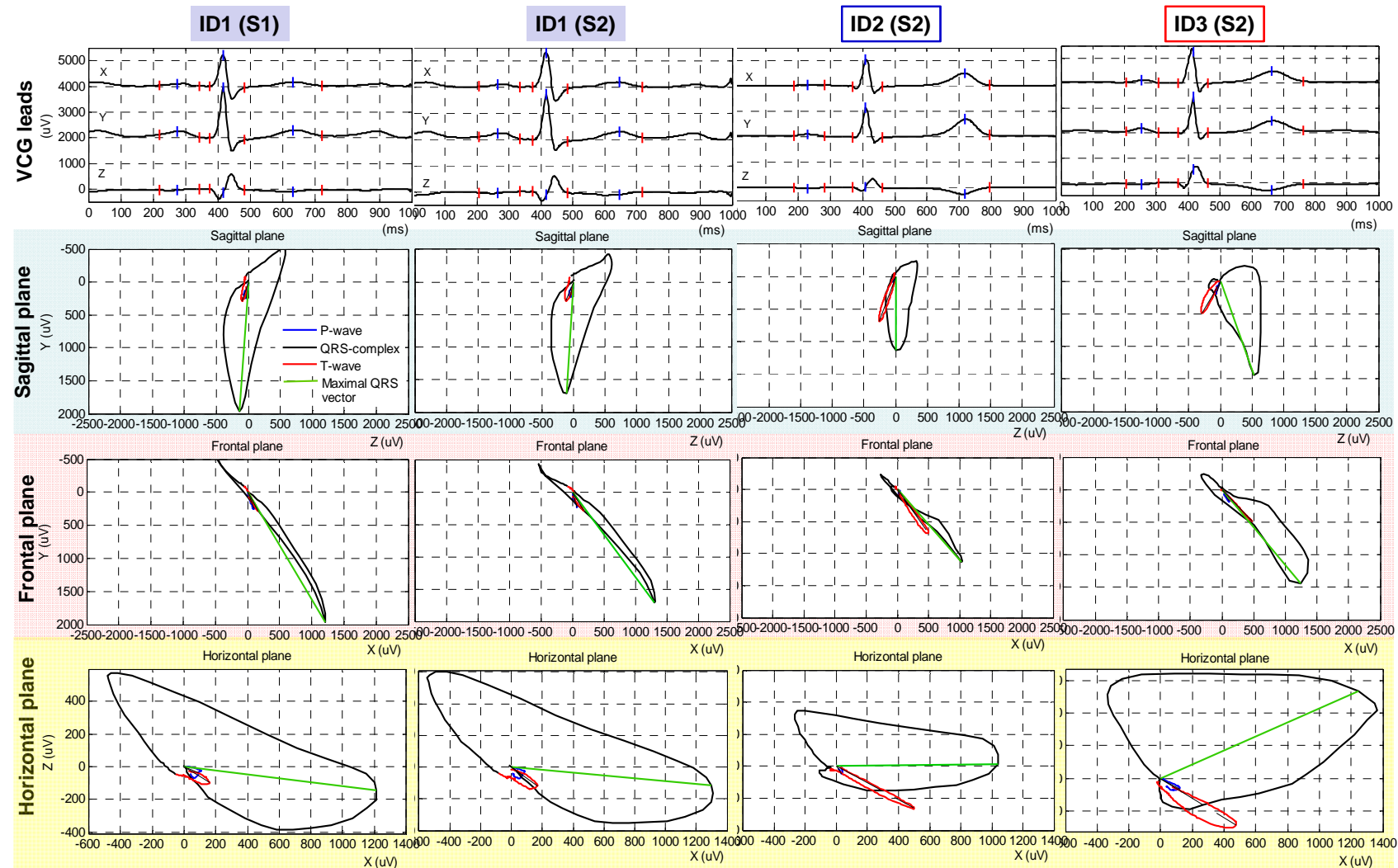

Figure 2. Example of VCG leads and 2D VCG loops obtained with Uijen inverse transformation of 12-lead ECG recordings from subject ID1 (S1 and S2 sessions) and subjects ID2, ID3 (S2 session). One column shows one session.

\section{Results and Discussion}

Figure 2 presents an example of the "who is who?" validation task, that should confirm if the $2^{\text {nd }}, 3^{\text {rd }}$ and $4^{\text {th }}$ recordings are from the subject of the $1^{\text {st }}$ recording. Although the 3 VCG leads (X, Y, Z) look quite similar and uncertain about the differences between all four recordings, the comparison of the VCG loops shows distinguishable differences for the $3^{\text {rd }}$ and $4^{\text {th }}$ recordings, e.g. QRS max vector azimuth is rotated (S, H-planes), QRS loop circumference, area and width are smaller ( $3^{\text {rd }}$ recording, S, F, H-planes) or larger $\left(4^{\text {th }}\right.$ recording, $\mathrm{H}-$ plane), T-loop is larger (S, F, H-planes).

Using an independent test dataset from a large population, we further report an unbiased assessment of different VCG-based human verification models. Figure 3 shows that 'Uijen' transformation has the top-scored TVR for all VCG features calculated in the F-plane (84.5\%), Splane (84.1\%), 3D-space (91.1\%), P-loop (70.9\%), QRSloop (89.6\%), T-loop (77.1\%) and all features (91.4\%), while both 'Dower' and 'Kors' transformations are topscored for H-plane (84.5\%), better by $0.7 \%$ than 'Uijen'. We distinguish the superiority of the 'Uijen' transformation with VCG synthesis predominantly from the limb leads (the largest Do coefficients).

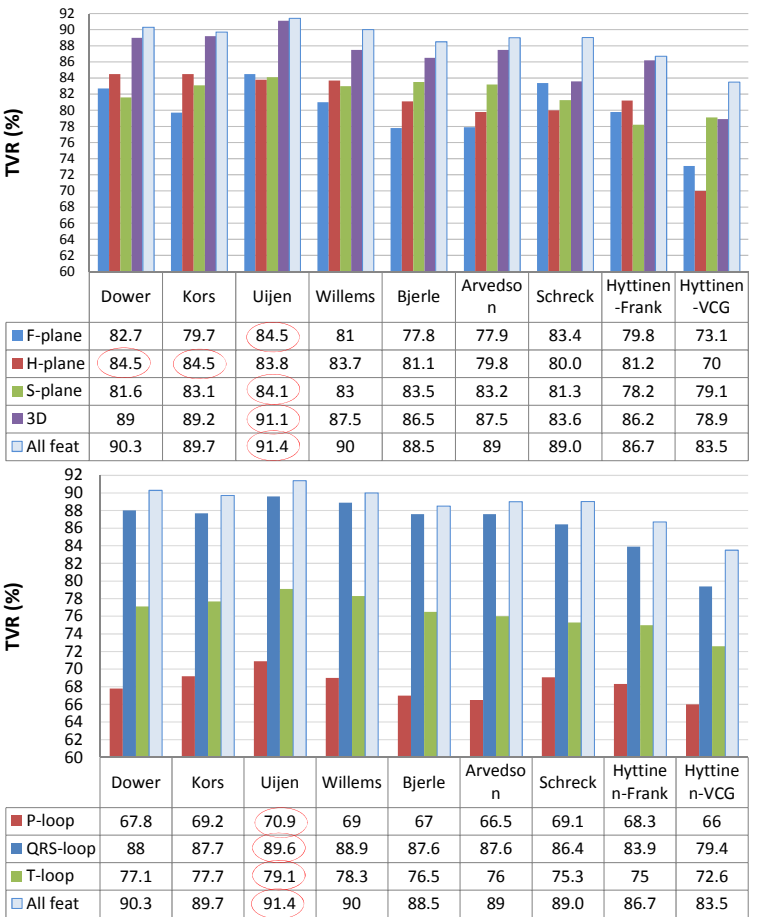

Figure 3. Comparison of different VCG transformations. The highest TVR for each feature set is highlighted. 


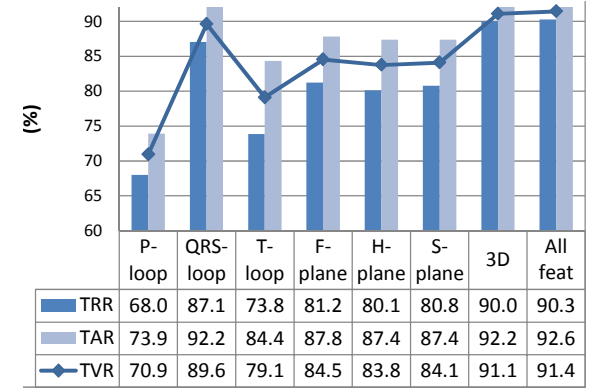

Figure 4. Validation of 'Uijen' transform on the test set.

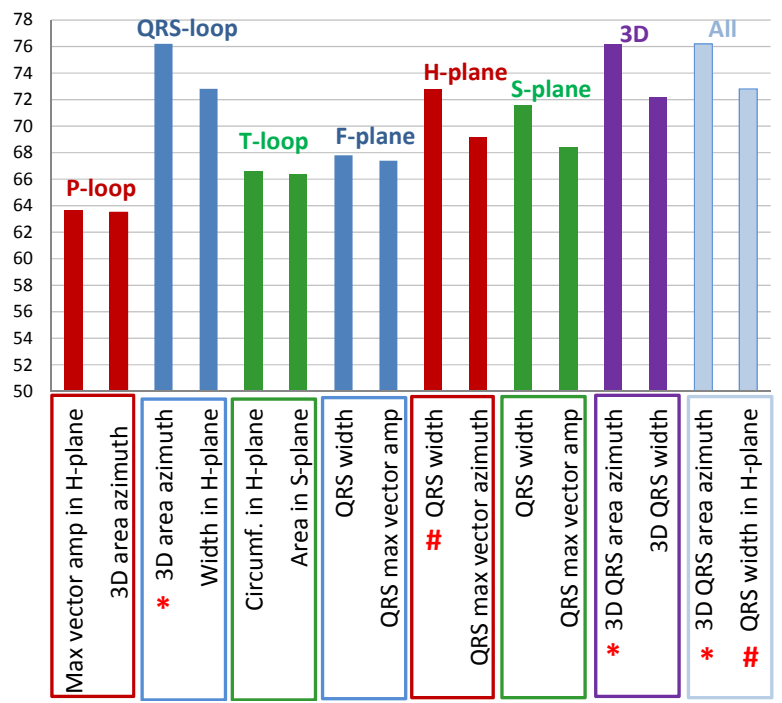

Figure 5. TVR(\%) of the top-scored single VCG features in loops and planes, using 'Uijen' transformation. Marks indicate the $1^{\text {st }}(*)$ and the $2^{\text {nd }}(\#)$ best features among all.

Taking the 'Uijen' transformation as a reference (Figure 4), the P-loop has the least biometric value with TVR about 7\% lower than T-loop and 19\% lower than QRS-loop. In fact, the QRS-loop carries the major biometric information (89.6\%) since P, T-loops could additionally increment TVR by merely $2 \%$. We find equal biometric significance of the three 2D planes (F, S, H about $84 \%$ ). 3D features carry almost full biometric information (91.1\%), only $0.3 \%$ lower than the model, including all features (91.4\%).

The VCG features with the most important individual impact for VCG-based biometrics are shown in Figure 5.

Using a clinically relevant database across a large population, representative of physiologically related longterm ECG changes and multi-session recording conditions, this study gives clear justification that VCG is applicable for human biometrics with TVR=83.5-91.4\% after testing of 9 established VCG transformations. We do not find VCG superiority in comparison to ECG-based human verification studies, showing $85.6-90.9 \%$ for single leads [3,4], 87.2-94.6\% for limb leads [5,7], 91.3\% for chest leads [7], 86-96.3\% for 12-lead ECG [6,7].

\section{Acknowledgements}

This study is supported by the Bulgarian Scientific Research Fund, grant T02/11.

\section{References}

[1] Odinaka I, Lai P, Kaplan A, O’Sullivan J, Sirevaag E, Rohrbaugh J. ECG biometric recognition: A comparative analysis. IEEE T Inf Foren Sec 2012;7(6):1812-24.

[2] Cimato S, Sassi R, Scotti F. Biometrics and privacy. Recent patents on Computer Science 2008;1:98-109.

[3] da Silva HP, Fred A, Lourenço A, Jain AK. Finger ECG signal for user authentication: Usability and performance. Proc. IEEE 6th Int Conf Biometrics 2013;1-8.

[4] Islam MS, Alajlan N. Biometric template extraction from a heartbeat signal captured from fingers. Multimed Tools Appl 2016;76(10):12709-33.

[5] Jekova I, Bortolan G. Personal verification/identification via analysis of the peripheral ECG leads. Influence of the personal health status on the accuracy. BioMed Research Internat 2015;135676.

[6] Jekova I, Krasteva V, Leber R, Schmid R, Twerenbold R, Müller C, et al. Intersubject variability and intrasubject reproducibility of 12-lead ECG metrics: Implications for human verification. J Electrocardiol 2016;49(6):784-9.

[7] Krasteva V, Jekova I, Abächerli R. Biometric verification by cross-correlation analysis of 12-lead ECG patterns: Ranking of the most reliable peripheral and chest leads. J Electrocardiol 2017 (in press)

[8] Abdelraheem M, Selim H, Abdelhamid T. Human identification using the main loop of the vectorcardiogram. Am J Signal Process 2012;2(2):23-9.

[9] Lee J, Cho B, Chee Y, Kim I, Kim S. A new approach for personal identification based on dVCG. IEICE T Inf Syst 2008;E91-D(4):1201-5.

[10] Lee J, Cho B, Kim I. Personal identification based on vectorcardiogram derived from limb leads electrocardiogram. J Appl Math 2012: 904905.

[11] Vozda M, Cerny M. Methods for derivation of orthogonal leads from12-lead electrocardiogram: A review. Biomed Signal Process Control 2015;19:23-34.

[12] Arvedson O. Methods for data acquisition and evaluation of electrocardiograms and vectorcardiograms with a digital computer. PhD Thesis, University of Umea, Sweden 1968.

[13] Belloch J, Guillem M, Climent A, Millet J, Hüsser D, Bollman A. Comparison of different methods for the derivation of the vectorcardiogram from the ECG and morphology descriptors. Comp in Cardiol 2007;34:435-8.

[14] Man S, Maan A, Schalij M, Swenne C. Vectorcardiographic diagnostic \& prognostic information derived from the 12-lead electrocardiogram: Historical review and clinical perspective. J Electrocardiol 2015;48:463-75.

Address for correspondence.

Vessela Krasteva

Institute of Biophysics and Biomedical Engineering, Acad. G. Bonchev str., bl.105, 1113, Sofia, Bulgaria, vessika@biomed.bas.bg 